\title{
Mutation screening of SEMA3A and SEMA7A in patients with congenital hypogonadotropic hypogonadism
}

\author{
Johanna Känsäkoski ${ }^{1,2}$, Rainer Fagerholm 1,3, Eeva-Maria Laitinen², Kirsi Vaaralahti 1,2, Peter Hackman ${ }^{4}$, Nelly Pitteloud ${ }^{5}$, \\ Taneli Raivio ${ }^{1,2}$ and Johanna Tommiska ${ }^{1,2}$
}

\begin{abstract}
BACKGROUND: Congenital hypogonadotropic hypogonadism $(H H)$, a rare disorder characterized by absent, partial, or delayed puberty, can be caused by the lack or deficient number of hypothalamic gonadotropin-releasing hormone $(\mathrm{GnRH})$ neurons. SEMA3A was recently implicated in the etiology of the disorder, and Sema7A-deficient mice have a reduced number of $\mathrm{GnRH}$ neurons in their brains.
\end{abstract}

METHODS: SEMA3A and SEMATA were screened by Sanger sequencing in altogether 50 Finnish $\mathrm{HH}$ patients (34 with Kallmann syndrome (KS; HH with hyposmia/anosmia) and 16 with normosmic $\mathrm{HH}(\mathrm{nHH})$ ). In 20 patients, mutation(s) had already been found in genes known to be implicated in congenital $\mathrm{HH}$.

RESULTS: Three heterozygous variants (c.458A>G (p.Asn153Ser), c.1253A>G (p.Asn418Ser), and c.1303G>A (p.Val435Ile)) were found in SEMA3A in three KS patients, two of which also had a mutation in FGFR1. Two rare heterozygous variants (c.442C $>$ T (p.Arg148Trp) and c.1421G $>$ A (p.Arg474G In)) in SEMA7A were found in one male $\mathrm{nHH}$ patient with a previously identified KISSIR nonsense variant and one male KS patient with a previously identified mutation in KAL1, respectively.

CONCLUSION: Our results suggest that heterozygous missense variants in SEMA3A and SEMATA may modify the phenotype of KS but most likely are not alone sufficient to cause the disorder.

\footnotetext{
ongenital hypogonadotropic hypogonadism (HH) is a rare disorder characterized by incomplete or absent puberty caused by the lack or deficient number of hypothalamic gonadotropin-releasing hormone $(\mathrm{GnRH})$ neurons, disturbed secretion or action of $\mathrm{GnRH}$, or both (1). Various patterns of inheritance have been observed in association with this condition, including X-linked, autosomal dominant, and autosomal recessive, as well as di- and oligogenic inheritance (2-4). Mutations in several genes, including KAL1, FGFR1,
}

FGF8, PROK2, PROKR2, CHD7, WDR11, GNRHR, GNRH1, KISS1R, KISS1, TAC3, and TACR3 $(5,6)$, have been shown to cause the disorder. In the majority of cases, however, the molecular genetic cause remains unresolved, implying the existence of additional genes underlying the condition (7). We have described the phenotypic and genotypic features of Finnish patients with Kallmann syndrome (KS) (8). A molecular genetic cause was obtained for $\sim 40 \%$ of Finnish KS patients, suggesting the presence of mutations in genes not yet implicated to underlie this syndrome.

Semaphorins are a class of secreted and membrane proteins that act as axonal growth cone guidance molecules. Sema3Aand Sema7A-mutant mice have a reduced number of $\mathrm{GnRH}$ neurons in their brains, with Sema3A being essential for the patterning of vomeronasal axons (9), whereas in Sema7A mutants, the olfactory system appears to remain unaffected (10). Thus, SEMA3A is a good candidate gene for KS and SEMA7A for normosmic $\mathrm{HH}$ (nHH). Two recent studies have suggested a role for $S E M A 3 A$ mutations in the pathogenesis of KS in humans $(11,12)$. However, studies on SEMA7A in patients with congenital HH have not yet been reported.

Our objective in the current work was to investigate whether mutations in these two candidate genes, SEMA3A and $S E M A 7 A$, are present in Finnish patients with congenital HH.

\section{RESULTS}

Overall, the mutation screening of SEMA3A and SEMA7A revealed few nonsynonymous variants. The nonsynonymous variants identified and the patients' phenotypes are presented in Table 1.

Among the $50 \mathrm{HH}$ patients, we identified three previously reported heterozygous missense variants, c.1253A $>\mathrm{G}$ (p.Asn418Ser), c.458A $>$ G (p.Asn153Ser), and c.1303G $>A$ (p.Val435Ile) in SEMA3A in three patients with KS. The patients with the Asn418Ser and Asn153Ser variants also had previously identified mutations in FGFR1:c.1305_1306dupAT (p.Ser436Tyrfs ${ }^{\star} 3$ ) and c.1825C $>\mathrm{T}$ (p.Arg609*), respectively 
(8). In the patient with the Val435Ile variant, no mutations in the known KS genes (KAL1, FGFR1, FGF8, PROK2, PROKR2, CHD7, WDR11, or NELF) were found. All the identified SEMA3A variants have been reported in the Exome Variant Server (EVS; NHLBI GO Exome Sequencing Project (ESP), Seattle, WA) database, with minor allele frequencies $0.03 \%$ (Asn418Ser), $0.5 \%$ (Asn153Ser), and $1.4 \%$ (Val435Ile) in the European American population (Table 1). The frequency of the identified variants was not determined in Finnish controls.

One heterozygous missense variant, c.442C $>\mathrm{T}$ (p.Arg148Trp), was found in SEMA7A in a male patient with congenital $\mathrm{nHH}$. The variant was predicted to be "probably damaging" by PolyPhen-2 (score: 1.00). A heterozygous nonsense variant, c.1167C>A (p.Cys389*) in KISS1R (NM_032551.4), a gene implicated in autosomal recessive $\mathrm{nHH}$, was identified previously in the same patient, but no other mutation was found when the coding region, promoter region, and the cDNA of KISS1R were Sanger sequenced. Neither of the mutations was present in 200 controls, and both were reported once in the EVS database: SEMA7A Arg148Trp 1/12986 and KISS1R Cys389* 1/10952 chromosomes. Another missense variant in SEMA7A, c.1421G>A (p.Arg474Gln), was identified in a KS patient who also had a KAL1 mutation deleting the last base of exon 8 and the first three bases of the following intron (g.2357_2360delAgta), thus disrupting a splice site (8). The Arg474Gln variant was predicted by PolyPhen-2 to be "probably damaging" with a score of 0.999 . The variant is absent in the EVS database, but several other amino acid substitutions have been reported in the same position (p.Arg474Leu, p.Arg474Pro, and p.Arg474Trp), suggesting that some variation is tolerated in this amino acid position.

\section{DISCUSSION}

KS patients with SEMA3A mutations were recently reported by two groups: Young et al. (11) identified a heterozygous deletion of $213 \mathrm{~kb}$ deleting 11 of the 17 exons in SEMA3A in a family with KS, whereas Hanchate et al. (12) identified one small deletion (Asp538fs*31) and several heterozygous missense variants in SEMA3A in KS patients. To further elucidate the contribution of SEMA3A mutations to the pathogenesis of KS, we investigated a cohort of well-characterized Finnish patients with $\mathrm{HH}$.

We identified three missense changes (Val435Ile, Asn153Ser, and Asn418Ser) in SEMA3A in three probands with KS. Two of these variants (Val435Ile and Asn153Ser) were also reported by Hanchate et al. (12). The Asn153Ser variant has been shown to cause reduced signaling activity in the GN11 cell line, whereas the Val435Ile variant results in impaired secretion of semaphorin $3 \mathrm{~A}$, suggesting that these two mutations have a pathogenic effect (12). Notably, two probands with SEMA3A variants (Asn153Ser and Asn418Ser) also had previously described truncating mutations in FGFR1 (8). Di-/oligogenic inheritance of $\mathrm{HH}$ has been continuously reported (2-4), and it seems possible that SEMA3A mutations might have a role in modifying the KS phenotype, although conclusive evidence is still lacking. For example, Young et al. (11) proposed that the heterozygous SEMA3A deletion might be sufficient to cause the disease phenotype because no other mutations were detected and the deletion cosegregated in the family with the apparently autosomal dominantly transmitted KS phenotype. On the other hand, Hanchate et al. (12) suggested that monoallelic mutations in SEMA3A are not sufficient to cause the disease phenotype based on the finding that all the missense variants they detected were reported in the EVS database, some were also found in healthy controls, and some of the patients also carried mutations in other genes implicated in KS. In none of the patients in our study, did mutations in SEMA3A seem to be the sole cause of the disorder as we only found heterozygous variants that are also present in healthy control individuals. The third patient, with the Val435Ile variant in SEMA3A and without other detected mutations in the known $\mathrm{HH}$ genes, most likely still has at least one unidentified mutation contributing to the disorder.

In the same vein, we wanted to screen in our patients another semaphorin gene, SEMA7A, which has recently been shown

Table 1. Clinical characteristics and molecular genetic findings in patients with $\mathrm{KS}$ or $\mathrm{nHH}$ with nonsynonymous variants in SEMA3A or SEMA7A

\begin{tabular}{|c|c|c|c|c|c|c|c|c|}
\hline Proband & Sex & Diagnosis & $\begin{array}{l}\text { Other phenotypic } \\
\text { features }\end{array}$ & $\begin{array}{c}\text { Family members with } \\
\text { KS or nHH }\end{array}$ & Gene & $\begin{array}{l}\text { Nucleotide and } \\
\text { amino acid change }\end{array}$ & $\begin{array}{l}\text { MAF } \\
(\%)^{\mathrm{a}}\end{array}$ & $\begin{array}{l}\text { Mutations in other } \\
\text { screened genes }^{\mathrm{b}}\end{array}$ \\
\hline 1 & M & KS & No & No & SEMA3A & $\begin{array}{l}\text { c.1303G>A (p.V435I), } \\
(\text { rs147436181) }\end{array}$ & 1.4 & Not detected \\
\hline 2 & $\mathrm{~F}$ & KS & $\begin{array}{l}\text { Cleft lip and dental } \\
\text { agenesis }\end{array}$ & $\begin{array}{l}\text { KS: sister, father, and } \\
\text { paternal aunt }\end{array}$ & SEMA3A & $\begin{array}{l}\text { c.458A>G (p.N153S), } \\
(\text { rs139295139) }\end{array}$ & 0.5 & $\begin{array}{l}\text { c.1825C>T (p.R609*) } \\
\text { (FGFR1), (rs121909639) }\end{array}$ \\
\hline 3 & $\mathrm{~F}$ & KS & No & KS: son and daughter & SEMA3A & $\begin{array}{l}\text { c.1253A>G (p.N418S), } \\
(\mathrm{rs142967103)}\end{array}$ & 0.03 & $\begin{array}{l}\text { c.1305_1306dupAT } \\
\text { (p.S436Yfs*3) (FGFR1) }\end{array}$ \\
\hline 5 & M & KS & $\begin{array}{l}\text { Micropenis, cryptorchidism, } \\
\text { and synkinesia }\end{array}$ & KS: maternal uncle & SEMA7A & c.1421G>A (p.R474Qc) & $\mathrm{N} / \mathrm{A}$ & $\begin{array}{l}\text { g.2357_2360delAgta } \\
(K A L 1)\end{array}$ \\
\hline
\end{tabular}

Rs-numbers of variants/mutations are given when available.

F, female; KS, Kallmann syndrome; $M$, male; MAF, minor allele frequency; $\mathrm{nHH}$, normosmic hypogonadotropic hypogonadism.

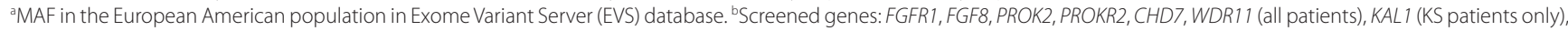
GNRHR, GNRH1, KISSIR, KISS1, TAC3, and TACR3 (nHH patients only). All mutations are heterozygous (except for the hemizygous mutation in KAL1). CNot found in the NCBI Short Genetic Variations database dbSNP or EVS database, but several other reported amino acid substitutions at the same position (p.R474L, p.R474P, and p.R474W). 


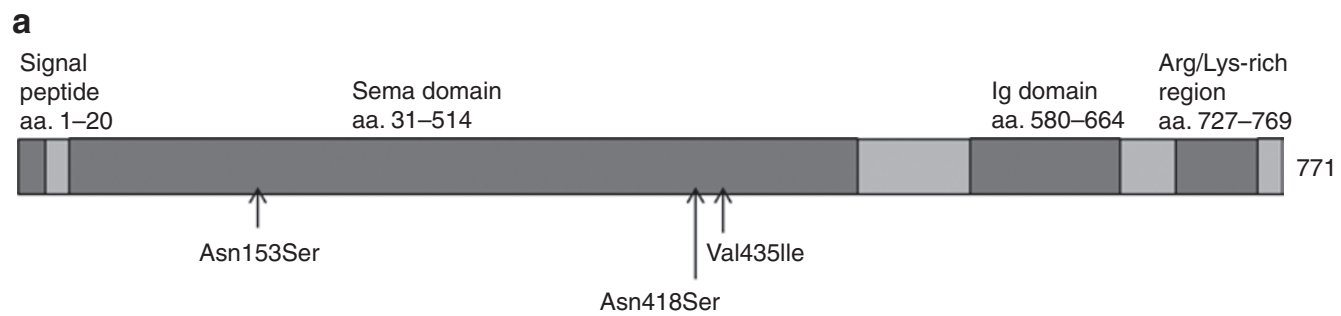

b

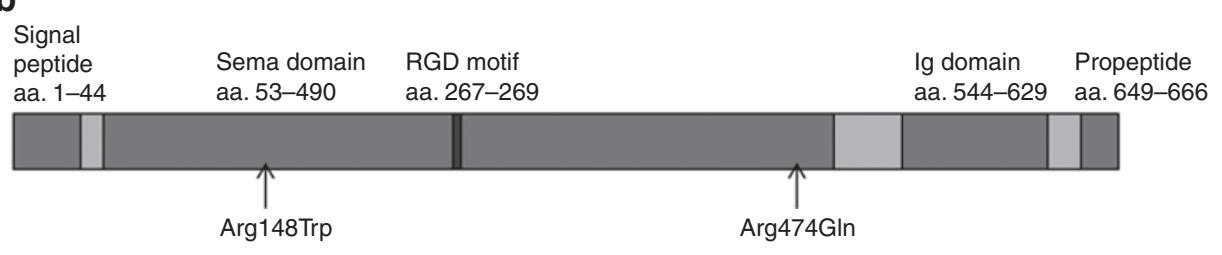

Figure 1. Functional domains of SEMA3A and SEMA7A and the identified variants. The named domains are shaded in darker gray. The predicted beginning and end of each domain according to UniProtKB protein knowledge database are indicated as amino acids (aa) below the name of the domain. (a) Functional domains of SEMA3A and the three identified missense variants. Arg/Lys-rich region, basic region rich in arginine and lysine; lg domain, Ig-like domain. (b) Functional domains of SEMA7A (isoform 1) and the two identified missense variants. RGD motif, Arginine-glycine-aspartate motif that is recognized by certain integrin receptors. The potential propeptide is cleaved in the mature form of the protein.

to have a role in the migration of GnRH neurons in mice (10). SEMA7A is able to promote axon outgrowth by signaling through integrin receptors containing the $\beta 1$ subunit (13) and promotes directional migration of $\mathrm{GnRH}$ neurons through $\beta 1$-integrin-dependent cell adhesion (10). $\beta 1$-integrin signaling was also recently shown to be involved in the development of GnRH neuron axonal projections to the median eminence in mice (14). Sema7 $A^{-/-}$adult mice have a $30 \%$ reduction of GnRH-1 cells in their brains compared with wild-type mice, and innervation of the median eminence by GnRH-1 neuron fibers is also clearly reduced (10). This phenotype seems to be the result of defective GnRH-1 neuron migration, which ultimately leads to reduced fertility in Sema $7 A^{-/-}$mice, whereas the development of the olfactory system appears to remain unaffected (10). We identified two rare variants in SEMA7A, both of which are predicted to be probably damaging by PolyPhen-2. Both of the variants are located in the conserved Sema domain of SEMA7A (Figure 1), which is responsible for receptor-binding specificity of the protein (15), although they are not located in the arginine-glycine-aspartate motif (amino acids 267-269) recognized by certain integrin receptors (16). It seems likely that the identified mutations alone are not sufficient to cause the disease phenotype, although they may contribute to it. Interestingly, one of the patients with a rare variant in SEMA7A also had a heterozygous nonsense mutation in KISS1R, a gene implicated in autosomal recessive $\mathrm{nHH}$ (17), bringing forth the possibility of di-/oligogenic inheritance. Whether or not this KISS1R variant is in homozygous or compound heterozygous state capable of causing $\mathrm{HH}$, however, is still unclear.

Taken together, the role of defective semaphorin signaling in $\mathrm{GnRH}$ deficiency has recently gained interest. Our results show that mutations in SEMA3A and SEMA7A are rare among Finnish patients but might have a role in modifying the disease phenotype. Further studies in different populations are warranted.

\section{METHODS}

Fifty patients with $\mathrm{HH}$ who were previously diagnosed on a basis of (i) clinical signs and symptoms of hypogonadism (absent puberty, infertility, and/or decreased libido), (ii) low sex steroid levels in association with inappropriately low or normal gonadotropin levels, and subnormal or normal response to GnRH stimulation test, (iii) otherwise normal anterior pituitary function, (iv) normal radiological imaging of the hypothalamic and pituitary areas, and in case of KS, (v) defective sense of smell detected by formal testing (UPSIT score $<5$ th percentile of age; University of Pennsylvania Smell Identification Test; Sensonic, Haddon Heights, NJ), and/or absent or rudimentary olfactory bulbs in magnetic resonance imaging, were screened for mutations in SEMA3A and SEMA7A. Patients were enrolled from all five university hospitals in Finland. Of them, 30 patients $(27$ men and 3 women), including $19 \mathrm{KS}$ patients (18 men and 1 woman), $9 \mathrm{nHH}$ patients ( 7 men and 2 women), and 2 men with adult onset $\mathrm{HH}$, were not found to have mutations in known $\mathrm{HH}$ genes $(8,18)$. In addition, $20 \mathrm{HH}$ patients were previously described to have mutation(s) in KAL1 (NM_000216.2; 3 patients), FGFR1 (NM_023110.2; 12 patients), GNRHR (NM_000406.2; 4 patients), or CHD7 (NM_017780.2; 1 patient) $(8,18-21)$.

The coding exons and exon-intron boundaries of SEMA3A (NM_006080.2) and SEMA7A (NM_003612.3) were PCR amplified from the genomic DNA of the patients. PCR products were purified with ExoSAP-IT treatment (Amersham Biosciences, Piscataway, $\mathrm{NJ}$ ) and bidirectionally sequenced using the ABI BigDyeTerminator Cycle Sequencing Kit (v3.1) and ABI Prism 3730xl DNA Analyzer automated sequencer (Applied Biosystems, Foster City, CA). The sequences were aligned and read with Sequencher 4.9 software (Gene Codes, AnnArbor, MI). All primer sequences and PCR conditions are available upon request. Exon 4 of SEMA7A was also sequenced in 200 controls. The minor allele frequencies of the identified variants were checked in the EVS database, and the effects of the identified SEMA7A variants were predicted with the web version of PolyPhen-2 software (22).

This study was performed with appropriate permissions from the Ethics Committee (E7) of the Helsinki University Central Hospital. Written informed consents were obtained from the participants, and also from their guardian if the participant was less than $16 \mathrm{y}$ of age.

\section{STATEMENT OF FINANCIAL SUPPORT}

This work was supported by the Academy of Finland, the Finnish Foundation for Pediatric Research, the Helsinki University Central Hospital Research Funds (EVO), Helsinki University Research Funds, the Sigrid Juselius Foundation, and Emil Aaltonen Foundation. 


\section{Articles | Känsäkoski et al.}

Disclosure: The authors declare that they have no competing interests.

\section{REFERENCES}

1. Seminara SB, Hayes FJ, Crowley WF Jr. Gonadotropin-releasing hormone deficiency in the human (idiopathic hypogonadotropic hypogonadism and Kallmann's syndrome): pathophysiological and genetic considerations. Endocr Rev 1998;19:521-39.

2. Pitteloud N, Quinton R, Pearce S, et al. Digenic mutations account for variable phenotypes in idiopathic hypogonadotropic hypogonadism. J Clin Invest 2007;117:457-63.

3. Falardeau J, Chung WC, Beenken A, et al. Decreased FGF8 signaling causes deficiency of gonadotropin-releasing hormone in humans and mice. J Clin Invest 2008;118:2822-31.

4. Sykiotis GP, Plummer L, Hughes VA, et al. Oligogenic basis of isolated gonadotropin-releasing hormone deficiency. Proc Natl Acad Sci USA 2010;107:15140-4.

5. Bianco SD, Kaiser UB. The genetic and molecular basis of idiopathic hypogonadotropic hypogonadism. Nat Rev Endocrinol 2009;5:569-76.

6. Brioude F, Bouligand J, Trabado S, et al. Non-syndromic congenital hypogonadotropic hypogonadism: clinical presentation and genotype-phenotype relationships. Eur J Endocrinol 2010;162:835-51.

7. Martin C, Balasubramanian R, Dwyer AA, et al. The role of the prokineticin 2 pathway in human reproduction: evidence from the study of human and murine gene mutations. Endocr Rev 2011;32:225-46.

8. Laitinen EM, Vaaralahti K, Tommiska J, et al. Incidence, phenotypic features and molecular genetics of Kallmann syndrome in Finland. Orphanet J Rare Dis 2011;6:41.

9. Cariboni A, Davidson K, Rakic S, Maggi R, Parnavelas JG, Ruhrberg C. Defective gonadotropin-releasing hormone neuron migration in mice lacking SEMA3A signalling through NRP1 and NRP2: implications for the aetiology of hypogonadotropic hypogonadism. Hum Mol Genet 2011;20:336-44.

10. Messina A, Ferraris N, Wray S, et al. Dysregulation of Semaphorin7A/ß1integrin signaling leads to defective GnRH-1 cell migration, abnormal gonadal development and altered fertility. Hum Mol Genet 2011;20:4759-74.
11. Young J, Metay C, Bouligand J, et al. SEMA3A deletion in a family with Kallmann syndrome validates the role of semaphorin $3 \mathrm{~A}$ in human puberty and olfactory system development. Hum Reprod 2012;27:14605.

12. Hanchate NK, Giacobini P, Lhuillier P, et al. SEMA3A, a gene involved in axonal pathfinding, is mutated in patients with Kallmann syndrome. PLoS Genet 2012;8:e1002896.

13. Pasterkamp RJ, Peschon JJ, Spriggs MK, Kolodkin AL. Semaphorin 7A promotes axon outgrowth through integrins and MAPKs. Nature 2003;424:398-405.

14. Parkash J, Cimino I, Ferraris N, et al. Suppression of $ß 1$-integrin in gonadotropin-releasing hormone cells disrupts migration and axonal extension resulting in severe reproductive alterations. J Neurosci 2012;32:169927002.

15. Tamagnone L, Comoglio PM. Signalling by semaphorin receptors: cell guidance and beyond. Trends Cell Biol 2000;10:377-83.

16. Hynes RO. Integrins: bidirectional, allosteric signaling machines. Cell 2002;110:673-87.

17. Seminara SB, Messager S, Chatzidaki EE, et al. The GPR54 gene as a regulator of puberty. N Engl J Med 2003;349:1614-27.

18. Laitinen EM, Tommiska J, Sane T, Vaaralahti K, Toppari J, Raivio T. Reversible congenital hypogonadotropic hypogonadism in patients with CHD7, FGFR1 or GNRHR mutations. PLoS ONE 2012;7:e39450.

19. Laitinen EM, Tommiska J, Dunkel L, Sankilampi U, Vaaralahti K, Raivio T. Idiopathic hypogonadotropic hypogonadism in a mother and her monozygotic twins born after a single embryo transfer. Fertil Steril 2010;93:2074. e13-6.

20. Laitinen EM, Hero M, Vaaralahti K, Tommiska J, Raivio T. Bone mineral density, body composition and bone turnover in patients with congenital hypogonadotropic hypogonadism. Int J Androl 2012;35:534-40.

21. Hero M, Tommiska J, Vaaralahti K, et al. Circulating antimüllerian hormone levels in boys decline during early puberty and correlate with inhibin B. Fertil Steril 2012;97:1242-7.

22. Adzhubei IA, Schmidt S, Peshkin L, et al. A method and server for predicting damaging missense mutations. Nat Methods 2010;7:248-9. 